\title{
Hydrazone crosslinked hyaluronan-based hydrogels for therapeutic delivery of adipose stem cells to treat corneal defects
}

\author{
Laura Koivusalo $^{\mathrm{a} \uparrow}{ }^{*}$, Jennika Karvinen $^{\mathrm{b} \dagger}$, Eetu Sorsa ${ }^{\mathrm{b}}$, Ilari Jönkkäri ${ }^{\mathrm{c}}$, Jari Väliaho ${ }^{\mathrm{b}}$, Pasi Kallio ${ }^{\mathrm{b}}$, Tanja \\ Ilmarinen $^{\mathrm{a}}$, Susanna Miettinen ${ }^{\mathrm{a}}$, Heli Skottman ${ }^{\mathrm{a}}$ and Minna Kellomäki ${ }^{\mathrm{a}} \mathrm{b}$ \\ ${ }^{a}$ BioMediTech Institute and Faculty of Medicine and Life Sciences, University of Tampere, Arvo \\ Ylpön katu 34, FI-33520 Tampere, Finland \\ ${ }^{\mathrm{b}}$ BioMediTech Institute and Faculty of Biomedical Sciences and Engineering, Tampere University of \\ Technology, Korkeakoulunkatu 3, FI-33101 Tampere, Finland \\ ${ }^{\mathrm{c}}$ Faculty of Engineering Sciences, Tampere University of Technology, FI-33101 Tampere, Finland, \\ Korkeakoulunkatu 6, FI-33101 Tampere, Finland \\ ${ }^{\dagger}$ Authors contributed equally \\ *Corresponding author
}

\begin{abstract}
Corneal blindness is a worldwide problem, plagued by insufficient amount of high-quality donor tissue. Cell therapy using human adipose stem cells (hASCs) has risen as an alternative to regenerate damaged corneal stromal tissue, the main structural and refractive layer of the cornea. Herein we propose a method to deliver hASCs into corneal defects in hyaluronan (HA)-based hydrogels, which form rapidly in situ with hydrazone crosslinking. We fabricated two different HA-based hydrazone-crosslinked hydrogels (HALD1-HACDH and HALD2-HAADH), and characterized their swelling, degradation, mechanical, rheological and optical properties and their ability to support hASC survival. To promote hASC attachment and survival, we incorporated collagen I (col I) to the more stable HALD1-HACDH hydrogel, since the HALD2-HAADH hydrogel suffered swift degradation in culture conditions. We then used an organ culture model with excised porcine corneas to study the delivery of hASCs in these three hydrogels for stromal defect repair. Although all hydrogels showed good hASC survival directly after encapsulation, only the collagen-containing HALD1-HACDH-col I hydrogel showed cells with elongated morphology, and significantly higher cell metabolic activity than the HALD1-HACDH gel. The addition of col I also increased the stiffness and reduced the swelling ratio of the resulting hydrogel. Most importantly, the corneal organ culture model demonstrated these hydrogels as clinically feasible cell delivery vehicles to corneal defects, allowing efficient hASC integration to the corneal stroma and overgrowth of corneal epithelial cells.
\end{abstract}

Keywords: hyaluronan, hydrogel, collagen I, adipose stem cells, cell delivery, corneal stroma 


\section{Introduction}

Corneal blindness due to trauma, burns and various inherited or acquired diseases is a worldwide problem, with estimated 1.5 to 2 million new cases annually [1]. Currently these cases are only treatable by transplantation of a donor cornea; a procedure restricted by immune reactions and graft failure, as well as a continuous shortage of suitable donor tissue [2]. The limitations of corneal transplants have driven the search for alternative treatment options, particularly by means of tissue engineering and stem cell therapy. Mesenchymal stem cells (MSCs) have gained great interest in corneal regeneration due to their immunomodulatory and antiangiogenic properties [3], as well as for their capability to inhibit corneal scarring [4]. Human adipose stem cells (hASCs) are an abundant and accessible source of adult MSCs [5], which have also been shown to differentiate towards corneal stromal keratocytes in vivo when delivered to the corneal stroma [6,7]. However, simple stromal injection of hASCs in saline solution results in only low amount of integrated cells and insufficient new collagen production [6,8], whereas hydrogel delivery increases the survival of hASCs in the corneal stroma [7].

Collagen I (col I) and hyaluronan (HA) are natural extracellular matrix (ECM) components, present in varying abundance in different tissues. $\mathrm{Col} \mathrm{I}$ is the main component of ECM in the corneal stroma, where it exists as highly regular fibrils for combined mechanical strength and high transparency [9]. HA is a high molecular weight polysaccharide, which has a high capacity to retain water and is degraded in vivo by hyaluronidase enzymes [10,11]. Rather than use HA in its native form, it can be modified through the carboxyl acid and hydroxyl groups in the D-glucuronic acid and N-acetyl-D-glucosamine sugar residues. HA-based hydrogels have also been previously suggested for corneal stromal repair, but to date they have required external crosslinkers and need to be preformed prior to implantation [7]. Hydrogel components, which gel upon mixing without external crosslinking agents, have the added advantage that they can be injected directly to the defect site where they are able to fill even irregularly shaped defects.

Hydrazone crosslinking has been widely used to prepare hydrogels for tissue engineering applications. Hydrazone crosslinking is a reaction between aldehyde- and hydrazide-groups and belongs to the group of pseudo click reactions (pseudo refers to moderate orthogonality). These reactions have many favorable properties, i.a. high reactivity, simple reaction conditions, no toxic reagents or side products, and high yields. [12] HA can be modified with complementary reactive aldehyde and hydrazide groups to enable this crosslinking. Aldehyde groups can be generated from vicinal diol groups of HA using periodate oxidation [13], or by incorporating an amino-glycerol side chain via an amidation reaction and selective oxidation of the pendent group of HA [14]. The latter method provides a less invasive way to modify the polymer. Hydrazide groups can be produced via reaction with either adipic acid dihydrazide [15,16] or carbodihydrazide [17]. The polyanionic behavior of HA at physiological $\mathrm{pH}$ hinders the adhesion of proteins and cells, which can be overcome by addition of other ECM binding 
sites, such as collagen [18]. Collagen can be incorporated to the previously described hydrogels, for example through imine formation, although it should be noted, that neutralized collagen can also form a gel on its own at $37^{\circ} \mathrm{C}$.

The aim of the study was to create transparent HA-based hydrogels for the delivery of hASCs for regeneration of the corneal stroma. In this study, we fabricated two HA-based hydrazone crosslinked hydrogels, and characterized their swelling, degradation, mechanical, rheological and optical properties and their ability to support hASC survival. We then further incorporated human col I into the more stable hydrogel, with the aim to promote hASC attachment and survival. In order to demonstrate proofof-concept, we used an organ culture model with excised porcine corneas to evaluate the clinical relevance of the HA-based hydrogels for hASC delivery to stromal defects.

\section{Materials and Methods}

\subsection{Materials and general methods}

Hyaluronic acid sodium salt $\left(\mathrm{M}_{\mathrm{w}}=1.5 \times 10^{5} \mathrm{~g} / \mathrm{mol}\right)$ was purchased from Lifecore (Chaska, MN, USA). Adipic acid dihydrazide (ADH), hyaluronidase from bovine testes (Type I-S, 400-1000 units/mg solid), hydroxylamine hydrochloride, acetic acid, sucrose, 1-hydroxybentzotriazole (HOBt), carbodihydrazide $(\mathrm{CDH}), 3$-amino-1,2-propanediol, t-butyl carbazate (TBC), picrylsulfonic acid solution $(5 \%(\mathrm{w} / \mathrm{v})$ in $\mathrm{H}_{2} \mathrm{O}$, TNBS), sodium cyanoborohydride, sodium periodate, sodium acetate, ethylene glycol, 1-Ethyl3-[3-(dimethylamino)propyl]carbodiimide (EDC), dimethyl sulphoxide (DMSO), and deuterium oxide (99.9 atom\% D, contains 0.05 wt.\% 3-(trimethylsilyl)-propionic-2,2,3,3-d4 acid, sodium salt) and collagen type I from human placenta were purchased from Sigma-Aldrich (St. Louis, MO, USA). Sodium chloride was purchased from J.T. Baker (Holland). All solvents used were of analytical quality. Milli-Q water was used in synthesis and determinations. Dialysis membranes (Spectra/Por ${ }^{\circledR}$ cut-off $3500,12-14,000$ and 25,000 g/mol) were purchased from Spectrum Laboratories, Inc. (Rancho Dominguez, CA, USA).

NMR-spectra were measured with Varian Mercury $300 \mathrm{MHz}$ NMR Spectrometer (Palo Alto, USA). Samples $(5 \mathrm{mg})$ were dissolved in deuterium oxide $(600 \mu \mathrm{L})$ containing internal standard $(0.05 \mathrm{wt} . \% 3$ (trimethylsilyl)-propionic-2,2,3,3-d4 acid, sodium salt). FTIR-spectra from hyaluronan components and formed hydrogels were measured on a Perkin Elmer Spectrum One ATR-FTIR Spectrometer (Waltham, MA, USA) in the spectral range of 400 to $4000 \mathrm{~cm}^{-1}$.

\subsection{Synthesis of aldehyde-modified hyaluronans}

Aldehyde groups were introduced to HA according to previously reported method [14]. Reaction scheme is shown in Fig. S1 (a). Briefly, HA (400 mg) was dissolved in deionized water (60 mL). 3amino-1,2-propanediol (182 mg), and HOBt (153 mg) pre-dissolved in 1:1 (v/v) mixture of acetonitrilewater $(2 \mathrm{~mL})$ were added, and $\mathrm{pH}$ of the solution was adjusted to $6(1 \mathrm{M} \mathrm{HCl})$. EDC $(58 \mathrm{mg})$ was added 
to the mixture under nitrogen and stirred overnight. Derivatized polymer was dialyzed with MW cutoff 3500 membrane against dilute $\mathrm{HCl}(\mathrm{pH} 3)$ containing $0.1 \mathrm{M} \mathrm{NaCl}$ for 48 hours and against dilute $\mathrm{HCl}$ (pH 3) for 24 hours. Purified polymer was lyophilized to obtain a white cotton-like product $(2,3-$ dihydroxypropyl amide derivative of HA). This product ( $200 \mathrm{mg}$ ) was dissolved in deionized water (25 $\mathrm{mL})$. Sodium periodate $(107 \mathrm{mg})$ pre-dissolved in deionized water $(0.5 \mathrm{~mL})$ was added to the solution in the dark at room temperature (RT) and stirred for 5 minutes. Ethylene glycol $(0.06 \mathrm{~mL})$ was added to inactivate unreacted periodate and solution was stirred for 2 hours. Derivatized polymer was dialyzed with MW cutoff 3500 membrane against deionized water for 24 hours. Purified polymer was lyophilized to obtain a white cotton-like product, HALD1. ${ }^{1} \mathrm{H}-\mathrm{NMR}\left(\mathrm{D}_{2} \mathrm{O}, 300 \mathrm{MHz}\right) 2,3-$ dihydroxypropyl amide derivative of HA: $\delta 4.53$ (br s, 1H), 3.83-3.34 (m, 10H), 2.00 (s, 3H). HALD1 (Fig. S3 (a), $\left.\mathrm{D}_{2} \mathrm{O}, 300 \mathrm{MHz}\right): \delta 9.57$ (s, 1H), 4.53 (br s, 1H), 3.65 (sharp s, 1H), 3.83-3.34 (m, 10H), $2.01(\mathrm{~s}, 3 \mathrm{H})$. FTIR (Fig. 1 (a), $\left.\mathrm{cm}^{-1}\right): 1732(v(\mathrm{C}=\mathrm{O})$ of $-\mathrm{C}(\mathrm{O}) \mathrm{H}), 1643(\mathrm{v}(\mathrm{C}=\mathrm{O})$ of sec. amide), 1617 $(\mathrm{d}(\mathrm{N}-\mathrm{H})$ of $-\mathrm{NHC}(\mathrm{O})-), 1558(\mathrm{~d}(\mathrm{~N}-\mathrm{H})$ of sec. amide).

Alternatively, a periodate oxidation was used to generate aldehyde groups from vicinal diol groups of HA according to previously reported method [13] with small modifications. Reaction scheme is shown in Fig. S1 (b). Briefly, sodium hyaluronate $(0.500 \mathrm{~g})$ was dissolved in deionized water $(100 \mathrm{~mL})$. Sodium periodate $(0.30 \mathrm{~g})$ was dissolved in deionized water $(2.7 \mathrm{~mL})$, added dropwise and stirred for 4 hours in the dark at RT under nitrogen. Ethylene glycol (4 equivalents) was added to inactivate any unreacted periodate and the solution was then stirred for 1 hour. Derivatized polymer was dialyzed with MW cutoff 25,000 membrane against deionized water for three days. Purified polymers were lyophilized to obtain white cotton-like product, HALD2. ${ }^{1} \mathrm{H}$ NMR (Fig. S3 (b), $\mathrm{D}_{2} \mathrm{O}, 300 \mathrm{MHz}$ ): HALD2: $\delta 4.47$ (m, 1H, H1 of glucose unit), 3.84-3.34 (m, 5H, H2-5 of glucose unit), 2.02 (s, 3H, NHC $(\mathrm{O}) \mathrm{CH} 3$ ). FTIR (Fig. 1 (b), $\left.\mathrm{cm}^{-1}\right)$ : HALD2: $1721(v(\mathrm{C}=\mathrm{O})$ of $-\mathrm{C}(\mathrm{O}) \mathrm{H}), 1633(v(\mathrm{C}=\mathrm{O})$ of $\mathrm{NHC}(\mathrm{O})$ - and $-\mathrm{C}(\mathrm{O}) \mathrm{OH}), 1618(\mathrm{~d}(\mathrm{~N}-\mathrm{H})$ of $-\mathrm{NHC}(\mathrm{O})-)$.

Degree of substitution (DS\%) of HALD components were determined with TNBS method similarly to $[19,20]$. Briefly, HALD (20 mg) was dissolved in acetate buffer ( $2 \mathrm{~mL}, 0.1 \mathrm{M}, \mathrm{pH} 5.2)$ and added to TBC solution in acetate buffer $(1 \mathrm{~mL}, 0.0348 \mathrm{~g}, 10$-fold excess per molar amount of sodium periodate used). The mixture was allowed to react for 1 hour at RT. Sodium cyanoborohydride $(1 \mathrm{~mL}, 0.0166 \mathrm{~g}$, equimolar amount to TBC) in acetate buffer was added and allowed to react for 24 hours at room temperature under nitrogen. The polymer was dialyzed with MW cutoff 25,000 membrane against 0.1 $\mathrm{M} \mathrm{NaCl}$ for 24 hours and for a further 24 hours in deionized water. Purified polymer was lyophilized to obtain a white cotton-like product. The ${ }^{1} \mathrm{H}$-NMR spectrum was measured and the DS\% was determined from the integration of ${ }^{1} \mathrm{H}-\mathrm{NMR}$ peaks. ${ }^{1} \mathrm{H}-\mathrm{NMR}\left(\mathrm{D}_{2} \mathrm{O}, 300 \mathrm{MHz}\right): \delta 2.0\left(3 \mathrm{H}, \mathrm{NHCOCH}_{3}\right)$ and $1.4(9 \mathrm{H}, \mathrm{t}-\mathrm{Boc})$. 


\subsection{Synthesis of hydrazide-modified hyaluronans}

Hydrazide groups were introduced to HA according to previously reported method [17]. Reaction scheme is shown in Fig. S1 (c). Briefly, HA (408 mg) was dissolved in deionized water $(100 \mathrm{~mL})$. Carbodihydrazide $(90 \mathrm{mg}$ ) and $\mathrm{HOBt}(153 \mathrm{mg})$ were added to the solution and $\mathrm{pH}$ was adjusted to 4.7 (0.1 M NaOH). EDC (19.17 mg) was added under nitrogen and stirred for overnight. Derivatized polymer was dialyzed with MW cutoff 3500 membrane against dilute $\mathrm{HCl}$ ( $\mathrm{pH} 3.5$ ) containing $0.1 \mathrm{M}$ $\mathrm{NaCl}$ for 48 hours and against deionized water for 24 hours. Purified polymer was lyophilized to obtain a white cotton-like product, HACDH. ${ }^{1} \mathrm{H}-\mathrm{NMR}$ (Fig. S4, $\mathrm{D}_{2} \mathrm{O}, 300 \mathrm{MHz}$ ): $\delta 4.54$ (br s, 1H), 3.84-3.34 (m, 10H), 2.02 (s, 3H). FTIR (Fig. 1 (a), $\left.\mathrm{cm}^{-1}\right): 1722$ and 1648 ( $\left.\mathrm{vC}=\mathrm{O}\right)$ of sec. amide), $1611(\mathrm{~d}(\mathrm{~N}-\mathrm{H})$ of prim. amine), $1557(\mathrm{~d}(\mathrm{~N}-\mathrm{H})$ of prim. amide), $1415(\mathrm{~d}(\mathrm{~N}-\mathrm{H})$ of sec. amide), $1028(\mathrm{v}(\mathrm{C}-\mathrm{N})$ of amine $)$.

DS\% of HACDH was determined with TNBS assay similarly to [17].

Alternatively, HA was modified with hydrazide groups via reaction with adipic acid dihydrazide. The fabrication of the polymer product, HAADH, has been previously reported in [16]. ${ }^{1} \mathrm{H}-\mathrm{NMR}$ and FTIR spectra are reported in the same article.

\subsection{Formation of hydrazone crosslinked hyaluronan-based hydrogels}

Hydrogels were fabricated according to Table 1 and Fig. S2. HALD1-HACDH hydrogel (H1) was prepared by dissolving the freeze dried HALD1 and HACDH components in PBS (pH 7.4) and mixing them in equal volumes. The gelation was shown to occur at RT and at $37{ }^{\circ} \mathrm{C}$; in this case the components were allowed to gel at $37{ }^{\circ} \mathrm{C}$ for at least $30 \mathrm{~min}$. HALD2-HAADH hydrogel (H2) was prepared similarly, with the freeze dried HALD2 and HAADH components dissolved in $10 \%$ sucrose. For different characterizations, cut syringes were used as molds to ease pushing the sample out. For cell culture, circular polydimethyl siloxane (PDMS) molds with $10 \mathrm{~mm}$ inner diameter were used. Solutions were sterilized prior to gelation using Whatman FP 30/0.2 CA-s $0.2 \mu \mathrm{m}$ (Whatman plc, Little Chalfont, UK) filters.

Additionally, human col I was introduced as a third gel component in H1-based hydrogel (H1C). Briefly, col I solution $(5 \mathrm{mg} / \mathrm{mL}$ in acetic acid, $\mathrm{pH} \approx 3$ ) was sonicated and then neutralized using $1 \mathrm{M}$ $\mathrm{NaOH}$. After neutralization, col I was mixed with HALD1 component followed by mixing with HACDH component. 
Table 1. Compositions of hydrazone crosslinked HA-based hydrogels.

\begin{tabular}{|l|l|c|c|c|}
\hline Code & \multicolumn{1}{|c|}{$\begin{array}{c}\text { Gel components } \\
\text { A-B-C }\end{array}$} & $\begin{array}{c}\text { DS } \% \\
\text { A / B }\end{array}$ & $\begin{array}{c}\text { Conc. } \mathbf{~ m g} / \mathbf{m L} \\
\text { A / B / C }\end{array}$ & $\begin{array}{c}\text { Volume ratio } \\
\text { A:B:C }\end{array}$ \\
\hline H1 & HALD1-HACDH & $15 / 17$ & $30 / 30$ & $1: 1$ \\
\hline H1C & HALD-HACDH-col I & $15 / 17$ & $30 / 30 / 5$ & $2: 2: 1$ \\
\hline H2 & HALD2-HAADH & $9 / 50$ & $20 / 10$ & $1: 1$ \\
\hline
\end{tabular}

The gelation time of hydrogels was determined using a tube tilt test [21], where the gel point can be determined simply by tilting a tube with gel solution and observing the time point where the system stops flowing.

\subsection{Swelling kinetics}

The swelling kinetics of the hydrogels were studied in cell culture medium (DMEM/F-12, Thermo Fisher Scientific, Waltham, MA, USA). Three parallel freshly made hydrogel samples (100 $\mu \mathrm{L})$ were weighed and placed into medium at $37^{\circ} \mathrm{C}$. At different time points, the hydrogels were weighed and the swelling ratio (SR) was calculated from the following equation:

$$
S R=\frac{W_{\text {swollen }}-W_{\text {initial }}}{W_{\text {initial }}} \times 100 \%
$$

where $\mathrm{W}_{\text {swollen }}$ is the mass of swollen hydrogel and $\mathrm{W}_{\text {initial }}$ is the mass of freshly made wet hydrogel.

\subsection{Enzymatic degradation}

The enzymatic degradation of the hydrogels was studied by incubating three parallel freshly made hydrogel samples $(100 \mu \mathrm{L})$ in hyaluronidase (HAse)-solution $\left(20-50 \mathrm{U} / \mathrm{mL}\right.$ in PBS) at $37{ }^{\circ} \mathrm{C}$ until they were completely degraded. At specific time points, the samples were weighed and the residual mass (\%) was calculated. Control samples, without hyaluronidase, were used to show that the degradation was not only due to hydrolysis.

\subsection{Rheological measurements}

The rheological measurements were performed using a rotational Haake RheoStress RS150 rheometer equipped with Rheowizard 4.3 software (ThermoHaake, Germany) with cone-plate geometry (20 mm diameter) and a gap of $0.8 \mathrm{~mm}$. Three parallel hydrogel samples $(500 \mu \mathrm{L}$, height $1 \mathrm{~mm} \times$ diameter 20 $\mathrm{mm}$ ) were prepared 24 hours before measurements into molds and covered with Parafilm. All rheological experiments were performed at $37{ }^{\circ} \mathrm{C}$ in the oscillatory mode. Amplitude sweep $(\gamma=0.01$ $10, \omega=1 \mathrm{~Hz})$ and frequency sweep $(\omega=0.1-10 \mathrm{~Hz}, \gamma=0.1)$ were used. Storage $\left(\mathrm{G}^{\prime}\right)$, loss $\left(\mathrm{G}^{\prime \prime}\right)$ and complex 
moduli $\left(\mathrm{G}^{*}\right)$ as well as the loss tangent $\left(\tan \delta=\mathrm{G}^{\prime \prime} / \mathrm{G}^{\prime}\right)$ indicating the overall viscoelasticity of material were determined [16].

\subsection{Mechanical measurements}

The compression measurements were conducted using a BOSE Electroforce Biodynamic 5100 machine equipped with a $225 \mathrm{~N}$ load sensor and Wintest 4.1 software (Bose Corporation, Eden Prairie, MN, USA). Five parallel hydrogel samples $(875 \mu \mathrm{L}$, height $7 \mathrm{~mm}$ x diameter $12 \mathrm{~mm})$ were prepared 24 hours before measurements into molds and covered with Parafilm. To keep the sample from slipping, the platen surfaces were covered with Parafilm. Samples were compressed using unconfined compression at a rate of $10 \mathrm{~mm} / \mathrm{min}$ to at least $65 \%$ strain in air at RT.

The stiffness of the materials was estimated based on the data obtained from a stress-strain curve. The determination was done similarly to [16]. Briefly, if the measured data is represented by a polynomial (6th order), the stiffness $(\Gamma)$ may be defined as a derivative of stress $(\sigma)$ with respect to strain $(\varepsilon)$ according to the equation:

$$
\frac{d \sigma}{d \varepsilon} \equiv \Gamma(\varepsilon)=\sum_{k=1}^{n} k c_{k} \varepsilon^{k-1}
$$

where the coefficients of the polynomial $c_{k}$ are the so-called elastic constants. Here, $c_{1}$ is the secondorder elastic constant, which is sometimes also called the elastic constant, Young's modulus or elastic stiffness constant [22-24]. Second-order elastic constants were estimated based on the means of stiffness polynomials of the five parallel samples at 0-0.15 strain with minimum standard deviations. It should be noted that the stiffness describes the same quantity as the second-order elastic constant, the latter being the stiffness at zero strain $\Gamma(0)=\mathrm{c}_{1}$.

\subsection{Optical measurements -refractive index and transparency}

Refractive indices of the hydrogels were determined using surface plasmon resonance equipment Navi 210A (BioNavis, Tampere, Finland). The device's goniometer and light source were used to scan the critical angle of total internal reflection between glass and hydrogel. A glass slide (BK7 glass) with a known refractive index was used for device calibration and measurements. After calibration, the flow cell of the device was filled with a hydrogel sample. A curve showing the reflection coefficient as a function of the angle of incidence was obtained from the measurement. The refractive indices of the hydrogels were determined from the curves utilizing Snell's law with the critical angle condition:

$$
n_{\text {gel }}=n_{\text {glass }} \sin (\alpha)
$$

where $n_{\text {gel }}$ and $n_{\text {glass }}$ are the refractive indices of hydrogel and glass slide ( $\left.n_{\text {glass }}=1.514,[25]\right)$, and $\alpha$ is the angle of incidence determined from the curve. 
The optical transparency of hydrogels was evaluated using a spectrophotometer (UV-VIS-NIR Spectrophotometer UV-3600Plus, Shimadzu, Kyoto, Japan) operating at the visible wavelength range (400-700 nm). Hydrogel samples $(900 \mu \mathrm{L}$, thickness $10 \mathrm{~mm}$ ) were prepared directly into the $1.5 \mathrm{~mL}$ semi-micro cuvettes. The hydrogel films (diameter $10 \mathrm{~mm}$ x thickness $1 \mathrm{~mm}$ ) were also photographed to show their optical clarity and transparency.

\subsection{Cell experiments in hyaluronan-based hydrogels}

\subsubsection{Cell encapsulation in hydrogels and in vitro cell culture}

This study was conducted under approval of the Ethics Committee of the Pirkanmaa Hospital District (Tampere, Finland, approval number R15161). The hASCs were isolated from adipose tissue samples from a female donor undergoing elective plastic surgery at Tampere University Hospital (Tampere, Finland) with patient's written consent. hASCs were isolated mechanically and enzymatically from subcutaneous adipose tissue as described previously $[26,27]$ and characterized for their surface marker expression by flow cytometry (FACSAria; BD Biosciences, Erembodegem, Belgium) as described in [27]. The cell characterization profile is shown in Table S1.

The hASCs were cultured in a medium containing Dulbecco's modified Eagle's medium/Ham's nutrient mixture F-12 (DMEM/F-12 1:1, Thermo Fisher Scientific, Waltham, MA, USA) supplemented with $5 \%$ human serum (type AB male, HIV tested from BioWest, Nuaillé, France), $1 \%$ L-glutamine (GlutaMAX ${ }^{\mathrm{TM}}$, Thermo Fisher Scientific) and $1 \%$ antibiotics $(100 \mathrm{U} / \mathrm{mL}$ penicillin, $100 \mu \mathrm{g} / \mathrm{mL}$ streptomycin, Lonza, Basel, Switzerland). The cells were maintained in T175 cell culture flasks at 37 ${ }^{\circ} \mathrm{C}$ in $5 \% \mathrm{CO}_{2}$, and passaged at approximately $80 \%$ confluence using TrypLE ${ }^{\mathrm{TM}}$ Select (Thermo Fisher Scientific).

Cells were encapsulated in hydrogels at passages 4-6. Prior to encapsulation, hASCs were detached from cell culture flasks and collected by centrifugation. Cells were resuspended in cell culture medium, counted and the appropriate number of cells required for each hydrogel were centrifuged to a pellet. Supernatant was removed and cell pellets were mixed by thorough pipetting into the aldehyde at a concentration of $4 \times 10^{6}$ cells $/ \mathrm{mL}$, with final hydrogel volume of $100 \mu \mathrm{L}$. The hydrogels were then formed in PDMS molds as described in Section 2.3. The cell-laden hydrogels were transferred onto tissue culture plates for further in vitro culture, with fresh hASC medium changed three times a week.

\subsubsection{Cell viability measurements}

The viability of hydrogel-encapsulated hASCs was assessed at time points 1 day, 3 days, 7 days and 10 days after encapsulation. Resazurin-based measurement of metabolic activity was performed using PrestoBlue $^{\circledR}$ Cell Viability Reagent and qualitative analysis of viable cells using LIVE/DEAD ${ }^{\circledR}$ viability/cytotoxicity kit for mammalian cells (both from Thermo Fisher Scientific). 
For PrestoBlue ${ }^{\circledR}$ analysis, three samples of each hydrogel containing hASCs and one sample without cells were washed once with DPBS (Lonza) and PrestoBlue ${ }^{\circledR}$ reagent diluted 1:10 (v/v) in hASC medium was added to the samples. After a 4-hour incubation at $37^{\circ} \mathrm{C}, 100 \mu \mathrm{L}$ aliquots of PrestoBlue ${ }^{\circledR}$ medium were collected in triplicate from each sample on a 96-well plate and their fluorescence was measured using Viktor 1420 Multilabel Counter (Wallac, Turku, Finland) at excitation and emission wavelengths of $544 \mathrm{~nm}$ and $590 \mathrm{~nm}$, respectively.

For qualitative analysis of cell viability, hydrogel samples were incubated with Live/Dead staining solution containing $2 \mu \mathrm{M}$ Calcein AM and $1 \mu \mathrm{M}$ Ethidium homodimer diluted in DPBS in $37^{\circ} \mathrm{C}$ for 45 min. The samples were washed with DPBS to reduce background fluorescence, and subsequently imaged using an Olympus IX51 fluorescence microscope equipped with a DP71 camera (Olympus Corporation, Tokyo, Japan).

\subsubsection{Cornea organ culture}

Whole porcine eyes were obtained from a local abattoir and kept on ice in AMES buffer (SigmaAldrich) containing $10 \mathrm{mM}$ HEPES and $2 \%$ antibiotics for up to 4 hours. Excess tissue was removed and the eyes were disinfected as described in [28]. The corneas were excised, moved to tissue culture plates, and cultured partially submerged in serum-free co-culture medium (CnT-Prime-CC, CellnTech, Bern, Switzerland) with $1 \%$ antibiotics, $0.25 \mu \mathrm{g} / \mathrm{mL}$ amphotericin B (Thermo Fisher Scientific) and 5 $\mu \mathrm{g} / \mathrm{mL}$ Plasmocin (InvivoGen, Toulouse, France) at $37{ }^{\circ} \mathrm{C}$ in $5 \% \mathrm{CO}_{2}$ for up to two weeks. The culture medium was changed three times a week, with dropwise addition of new medium to wet the central cornea.

For implantation of hydrogels to porcine corneas, they were mounted on a Barron artificial anterior chamber (Katena products Inc., Denville, NJ, USA) where the operation was performed. First, the corneal epithelium was scraped off using the flat edge of a scalpel (Feather Safety Razor co,. Itd, Osaka, Japan). Then, a 5-mm trephine (Robbins Instruments, Chatham, NJ, USA) was used to make a partial depth incision in the center of the cornea, from which the stroma was removed with a crescent knife (Bausch\&Lomb Inc, Rochester, NY, USA). In the formed stromal cavities, hydrogels with hASCs ( $\mathrm{n}=$ $5)$ and without any cells $(n=2)$ were cast as described in Sections 2.3. and 2.10. with final hydrogel volume of $50 \mu \mathrm{L}$. After gelation, the corneas were removed from the artificial anterior chamber, placed back into culture plates and covered with silicone contact lenses (EyeQ One-Day Premium, Cooper Vision, Hamble, UK). The culture medium was changed to hASC medium, with the corneas partially submerged. The porcine corneas were cultured for 7 days with the hydrogels and subsequently fixed for 4 hours in RT with either $4 \%$ PFA or acid-formalin/EtOH -fixative $(10 \%$ formaldehyde in $70 \%$ ethanol and $5 \%$ glacial acetic acid, all v/v), as the acid-formalin/EtOH should preserve HA better than the standard formalin fixation [29]. The samples were dehydrated in an automated tissue processor (Tissue-Tek VIP, Sakura Finetek Europe) and embedded in paraffin. 
To evaluate hASC incorporation into the corneal stroma, immunohistochemical staining for the panhuman marker TRA-1-85 was performed. 5- $\mu$ m-thick sections of samples were mounted onto SuperFrost glass slides, conventionally deparaffinated and hydrated. Antigen retrieval was performed by placing slides in hot $0.01 \mathrm{M}$ citrate buffer ( $\mathrm{pH}$ 6.0) for $10 \mathrm{~min}$. After cooling to RT, samples were washed with PBS, endogenic tissue peroxidases were inactivated with $3 \% \mathrm{H}_{2} \mathrm{O}_{2}$ for $5 \mathrm{~min}$, and unspecific binding sites were blocked by incubation with $2.5 \%$ normal horse serum (Vector ImmPress reagent, Vector Laboratories) at $37{ }^{\circ} \mathrm{C}$ for $45 \mathrm{~min}$. Samples were subsequently incubated with antiTRA-1-85 mouse IgG antibody (courtesy of Peter Andrews, University of Sheffield) in a 1:50 (v/v) dilution in $0.5 \% \mathrm{BSA}$ at $37^{\circ} \mathrm{C}$ for $60 \mathrm{~min}$, and washed with PBS. Secondary labeling with the reporter enzyme was done by incubating samples with Vector ImmPress Reagent (Vector Laboratories, Inc., Burlingame, CA, USA) for 30 min at RT. After subsequent washes in PBS, peroxidation was performed for 5 min at RT with Dako liquid DAB+chromogen system (Dako North America, Inc., Carpinteria, CA, USA). Finally, samples were counterstained with Harris hematoxylin (Millipore, Billerica, MA, USA) and mounted with Pertex (Histolab, Askim, Sweden). Samples were imaged with a Nikon Eclipse TE2000-S microscope equipped with a DS-Fi1 camera (Nikon Instruments, Amsterdam, Netherlands).

\subsection{Statistical data analysis}

Statistical data analyses were performed with MATLAB (Statistics and Machine Learning Toolbox ${ }^{\mathrm{TM}}$ ). All the quantitative data are presented as mean and standard deviation. A non-parametric KruskalWallis test and a Wilcoxon rank sum test were used to determine whether there were statistically significant differences within the mechanical and rheological data set, and to analyze specific sample pairs, respectively. Due to a relatively low $n$, a non-parametric testing was chosen. Bonferroni correction was used when more than two groups were compared. A p-value $<0.05$ was considered significant.

\section{Results and Discussion}

\subsection{Synthesis of complementary reactive hyaluronan components}

Hyaluronans were modified with aldehyde groups either through periodate oxidation or through selective oxidation of diol-modified HA. The modifications were confirmed with ${ }^{1} \mathrm{H}-\mathrm{NMR}$ and FTIR analysis. In the ${ }^{1}$ H-NMR spectrum (Fig. S3 (a)) of HALD1, a barely observable aldehyde peak (9.57 ppm) was detected, whereas with HALD2 it was not visible (Fig. S3 (b)). The reason why the aldehyde peak is not usually observed, is due to a reversible hemiacetal formation [30]. The presence of aldehyde-groups was confirmed based on the FTIR-spectrum (Fig. 1), where the typical aldehyde shoulder (HALD1: $1732 \mathrm{~cm}^{-1}$, HALD2: $1721 \mathrm{~cm}^{-1}$ ) was detected. The benefit of using selective oxidation of diol-modified HA instead of periodate oxidation is that it keeps the ring-structure of HA intact and therefore HA is more easily recognized by the cells. Hyaluronans were also modified with hydrazide-groups using either $\mathrm{CDH}$ or $\mathrm{ADH}$ as a source of the hydrazide unit. The ${ }^{1} \mathrm{H}-\mathrm{NMR}$ spectrum 
(Fig. S4) of HACDH hardly differed from that of non-modified HA (spectrum not shown), whereas in the FTIR-spectrum (Fig. 1 (a)) signals from the hydrazide unit were detected, including strong amide $\mathrm{C}=\mathrm{O}$ stretching $\left(1722 \mathrm{~cm}^{-1}\right)$ and amide N-H deformation $\left(1611\right.$ and $\left.1557 \mathrm{~cm}^{-1}\right)$ signals. The benefit of using $\mathrm{CDH}$ instead of $\mathrm{ADH}$ is discussed more deeply in the next section. The DS\% of HALD1 and HACDH components were kept relatively low not to lose the favorable properties of original polymer (Table 1.).

(a)

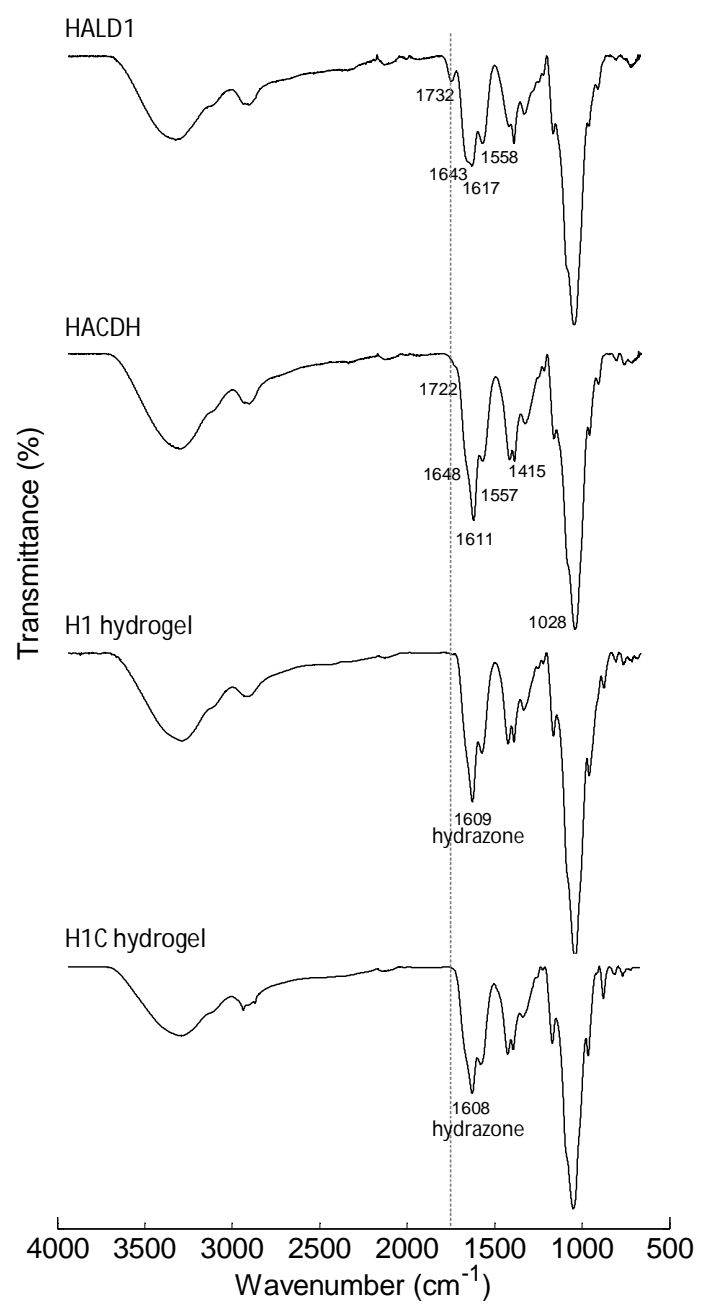

(b)



Fig. 1. Chemical structure analysis of polymer components and HA-based hydrogels. (a) FTIR spectra of HALD1 and HACDH components and hydrazone crosslinked HALD1-HACDH(-col I) hydrogels (H1 and H1C). (b) FTIR spectra of HALD2 and HAADH components and hydrazone crosslinked HALD2-HAADH (H2) hydrogel. The appearance of a hydrazone $\mathrm{C}=\mathrm{N}$ stretching signal at 1609, 1608 and $1606 \mathrm{~cm}^{-1}$ for $\mathrm{H} 1, \mathrm{H} 1 \mathrm{C}$ and $\mathrm{H} 2$ hydrogels (respectively) and the disappearance of an aldehyde signal at $1732 \mathrm{~cm}^{-1}$ for $\mathrm{H} 1$ and $\mathrm{H} 1 \mathrm{C}$ hydrogels, and at $1721 \mathrm{~cm}^{-1}$ for $\mathrm{H} 2$ hydrogel confirmed the presence of hydrazone crosslinking. 


\subsection{Formation of hydrazone crosslinked hydrogels}

HA-based hydrazone crosslinked hydrogels were formed from complementary reactive HA components according to Table 1 and Fig. S2. Different aldehyde- and hydrazide-modified components were used for the two hydrogel types, $\mathrm{H} 1$ and $\mathrm{H} 2$. H1 hydrogel was composed of the HALD1 component formed by selective oxidation of diol-modified HA, and the HACDH component formed by reacting the carboxylic groups in $\mathrm{HA}$ with an excess of $\mathrm{CDH}$ in the presence of $\mathrm{EDC}$ and $\mathrm{HOBt}$. $\mathrm{H} 2$ hydrogel, on the other hand, was composed of the periodate oxidized HALD2 component and the HAADH component formed by reacting the carboxylic groups in HA with an excess of ADH in the presence of EDC and HOBt. H1 hydrogel was considered more stable due to a resonance stabilization effect of $\mathrm{CDH}$ [17]. $\mathrm{CDH}$ has a neighboring heteroatom $\left(\mathrm{N}^{3}\right)$ providing resonance stabilization to the developing $\mathrm{N}^{2}$ positive charge. This type of linker $\left(\mathrm{C}^{1}=\mathrm{N}^{1}-\mathrm{N}^{2} \mathrm{H}-(\mathrm{C}=\mathrm{O}) \mathrm{N}^{3} \mathrm{H}\right)$ allows the delocalization of $\mathrm{N}^{2}$ positive charge due to its urea-type structure. There is no stabilization effect with ADH. [17] H1 hydrogel was preferred due to the more stable structure and the less invasive modification of HALD1 component, thus the effect of col I addition was only tested with the H1 hydrogel. The gelation of $\mathrm{H} 1$ hydrogels was successful only when PBS or cell culture medium solutions were used, whereas H2 hydrogel gelated only in deionized water or in $10 \%$ sucrose. Sucrose was used to make the osmotic pressure more suitable for the cells in cell culture experiments, therefore it was used in other experiments too.

The hydrogels gelated in 3-5 minutes, therefore it was possible to mix the components, for example by pipetting the gel solution few times back and forth in the tip. Alternatively, a double syringe system could be used, making the hydrogel injectable. The gelation time is appropriate for clinical applications of the hydrogels as a cell delivery vehicle, as the gels can flow to fill the wound site, but gelate rapidly enough for a fast and simple implantation.

The chemical structure of the hydrogels was determined with FTIR. The FTIR spectra (Fig. 1) of hydrogels showed the appearance of a hydrazone $\mathrm{C}=\mathrm{N}$ stretching signal at 1609,1608 and $1606 \mathrm{~cm}^{-1}$ for $\mathrm{H} 1, \mathrm{H} 1 \mathrm{C}$ and $\mathrm{H} 2$ hydrogels and the disappearance of an aldehyde signal at $1732 \mathrm{~cm}^{-1}$ for $\mathrm{H} 1$ and $\mathrm{H} 1 \mathrm{C}$ hydrogels, and at $1721 \mathrm{~cm}^{-1}$ for $\mathrm{H} 2$ hydrogel.

\subsection{Swelling kinetics}

The swelling tests were conducted in cell culture medium (DMEM/F-12) to determine hydrogel swelling behavior in the cell culture environment. The swelling ratios (SR, \%) of the hydrogels are shown in Fig. 2 (a). The results revealed a difference in the stability of hydrogels. Both H1 and H1C hydrogels were stable even after 48 hours, whereas $\mathrm{H} 2$ hydrogel degraded in hours, and after 24 hours there was no gel left to weigh. Both $\mathrm{H} 1$ and H1C hydrogels swell considerably. After 48 hours, the SR of $\mathrm{H} 1$ hydrogel was over $200 \%$, whereas that of H1C hydrogel was approximately $150 \%$. Although the SRs are quite high considering the final application, it is notable that the addition of col I lowered 
the SR approximately $20 \%$ at each data point. One possible explanation for this is that the imine formation between amino groups of collagen and the aldehyde groups of HA makes the structure of $\mathrm{H} 1 \mathrm{C}$ hydrogel more crosslinked. Higher degree of crosslinking reduces the swelling ratio. There is also a marked difference in the swelling behavior of the components themselves; HA is susceptible to swelling whereas collagen is not. As expected, replacing some of the HA with collagen led to a lower swelling ratio.

(a)



(b)

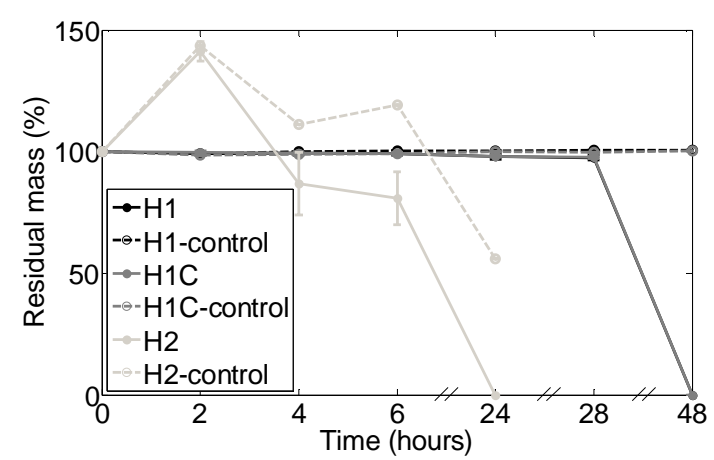

Fig. 2. Swelling kinetics and enzymatic degradability of HA-based hydrogels. (a) Swelling ratio (SR, $\%$ ) as a function of time (hours) curves of HALD1-HACDH (H1), HALD1-HACDH-col I (H1C) and HAL2-HAADH (H2) hydrogels in cell culture medium (DMEM/F-12). (b) Enzymatic degradation profiles of $\mathrm{H} 1, \mathrm{H} 1 \mathrm{C}$ and $\mathrm{H} 2$ hydrogels in HAse enzyme (20-50 U/mL in PBS). Both experiments showed the instability of $\mathrm{H} 2$ hydrogel, whereas $\mathrm{H} 1$ and $\mathrm{H} 1 \mathrm{C}$ were shown to be more stable. The mean $(\mathrm{n}=3)$ and standard deviation bars are shown.

Usually the swelling ratio is determined by studying the water uptake of dry hydrogels. In this case, it was more informative to study the swelling of freshly made wet hydrogels, because this form of gel was used in the cell culture experiments. It is also known that the drying methods, for example freezedrying, affect the hydrogel structure [31] and therefore can distort the swelling results. We have also noticed this in our studies (data not shown).

\subsection{Enzymatic degradation}

Enzymatic degradation of the hydrogels was studied using HAse enzyme (20-50 U/mL in PBS). The degradation curves are shown in Fig. 2 (b). The results showed that the degradation behavior of $\mathrm{H} 1$ and $\mathrm{H} 2$ hydrogels was very different. $\mathrm{H} 1$ and $\mathrm{H} 1 \mathrm{C}$ hydrogels remained relatively stable for 28 hours, after which they were completely degraded by the enzyme by the 48 -hour time point. H1C hydrogel was slightly more stable than H1, but not significantly. The H1 and H1C control hydrogels did not exhibit significant degradation during the 48 hours, indicating that the observed degradation is truly enzymatic rather than caused by simple hydrolysis. On the other hand, $\mathrm{H} 2$ hydrogel degraded in a matter of hours. After 24 hours, there was no gel left to weigh. Even the control hydrogel started to degrade at the same 
time, which refers to its tendency to simple hydrolysis caused by the solution environment. Similar behavior was observed in the swelling tests. Different stability behavior between the H1, H1C and H2 hydrogels can be explained by the resonance stabilization effect of CDH described earlier (Section 3.2.).

Based on the results, HAse which degrades HA in biological systems, can recognize the modified HA components and therefore indicates that these hydrogels should be degradable also in vivo. HA degradation products are mainly metabolized in the lymph nodes and liver, or locally in alymphatic, densely structured tissues, such as the cornea [10]. As cultured tissue finally replaces the hydrogel scaffold material, the degradation rate of these hydrogels should be tested more thoroughly in actual conditions in order to see if it matches the regeneration rate of the receiving native tissue, as the true HAse enzyme concentration in the cornea may vary from the amount of enzyme used here. It should be noted that the remodeling process of the injured stroma can take months or even years to complete [32].

\subsection{Rheological properties}

The viscoelastic properties of the hydrogels were determined using rheological tests. The amplitude dependence of modulus for hydrogels is presented in Fig. 3 (a). The linear viscoelastic region (LVR) was determined for all the hydrogels. The hydrogels showed linear behavior of G' up to about $10 \%$ strain. Outside this region, $\mathrm{G}^{\prime}$ dropped indicating a structure breakdown.

The frequency sweep measurements were performed based on the LVR. The frequency dependence of modulus is presented in Fig. 3 (b). A true gel structure was discovered, meaning that $G^{\prime}$ was parallel to G". Moreover, G' was higher than G" and independent of frequency, which is typical for ideal gels. [33] The $\mathrm{H} 1$ and $\mathrm{H} 1 \mathrm{C}$ hydrogels showed more linear behavior of $\mathrm{G}^{\prime}$ and $\mathrm{G}^{\prime \prime}$ than the $\mathrm{H} 2$ hydrogel. Especially, G" of the H2 hydrogel was more non-linear, indicating possible non-homogeneity of the structure. This can cause changes in the structure during the measurement. For the H2 hydrogel, the G" increased at higher strains. This means that there is less time for greater movement of the polymer chains and polymer chains fail to rearrange at a given time scale. This will lead to stiffening and more solid-like behavior. [34]

The complex modulus $\left(\mathrm{G}^{*}\right)$ values of the hydrogels are presented in Table 2. The $\mathrm{G}^{*}$ of the $\mathrm{H} 1$ and $\mathrm{H} 1 \mathrm{C}$ hydrogels were higher than with the $\mathrm{H} 2$ hydrogel, although there were no statistically significant differences between the gels. Higher $\mathrm{G}^{*}$ indicates stiffer structure and higher resistance to deformation. It is also known that if the loss tangent is lower than 0.1 , the hydrogels are considered as strong gels. [35] This was true for all the tested hydrogels $(\mathrm{H} 1: \tan \delta=0.084, \mathrm{H} 1 \mathrm{C}: \tan \delta=0.096$ and $\mathrm{H} 2: \tan \delta=$ $0.014)$. 
(a)

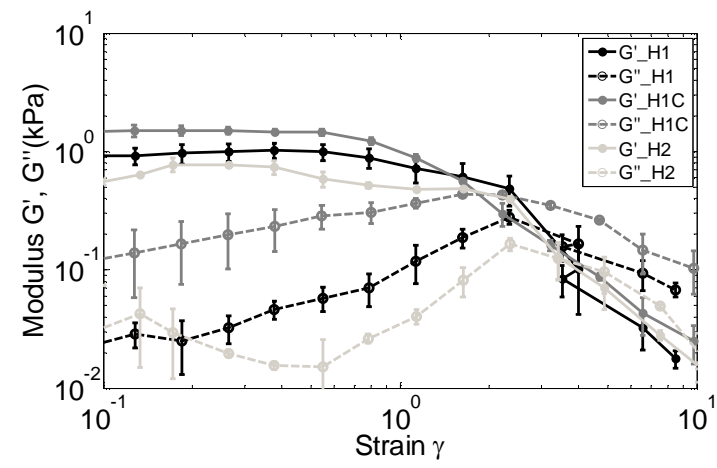

(c)

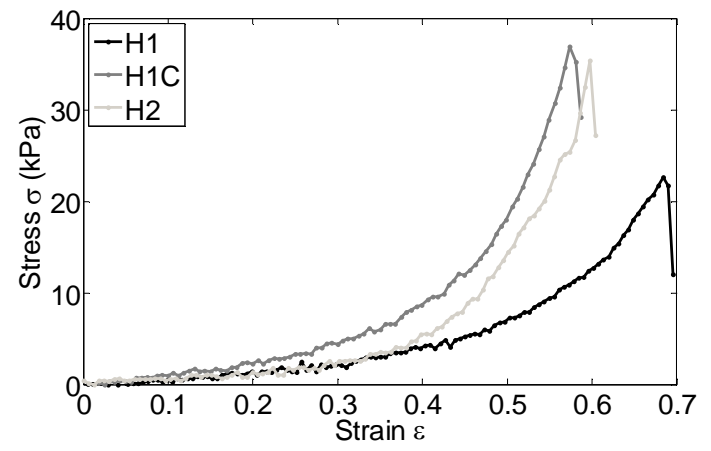

(b)

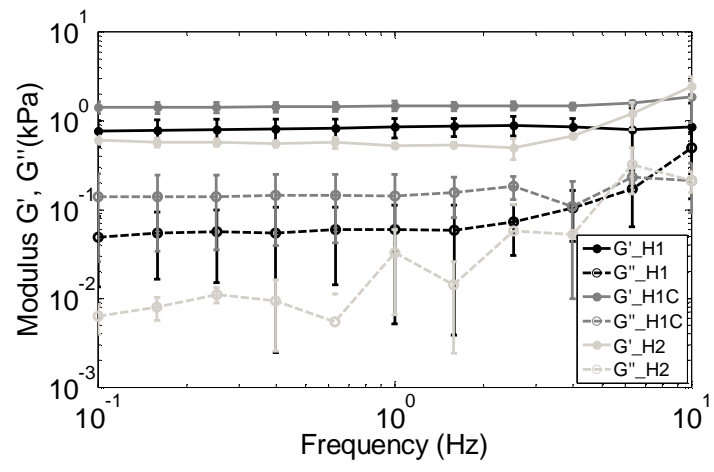

(d)



Fig. 3. Rheological and mechanical properties of HA-based hydrogels. (a) Amplitude dependence and (b) frequency dependence of modulus of HALD1-HACDH (H1), HALD1-HACDH-col I (H1C) and HALD2-HAADH (H2) hydrogels (measured with Haake RheoStress RS150 rheometer). The mean (n $=3$ ) and standard deviation are shown. (c) Stress as a function of strain (representative curves, measured with BOSE Electroforce Biodynamic 5100 machine equipped with a $225 \mathrm{~N}$ load sensor), and (d) stiffness as a function of strain (average curves, calculated similarly to [16]) curves of H1, H1C and H2 hydrogels.

Table 2. The complex modulus, second-order elastic constant, and refractive index values of HALD1HACDH (H1), HALD1-HACDH-col I (H1C) and HALD2-HAADH (H2) hydrogels. * p < 0.05 H1C with respect to $\mathrm{H} 2$. There were no statistically significant differences found between the complex modulus of hydrogels.

\begin{tabular}{|l|c|c|c|}
\hline Gel & $\begin{array}{c}\text { Complex modulus, G* } \\
\mathbf{k P a}\end{array}$ & $\begin{array}{c}\text { Second-order elastic constant } \\
\mathbf{k P a}\end{array}$ & Refractive index \\
\hline H1 & $0.81 \pm 0.23$ & $6.7 \pm 1.0$ & 1.337 \\
\hline H1C & $1.42 \pm 0.16$ & $11.6 \pm 1.7^{*}$ & 1.334 \\
\hline H2 & $0.57 \pm 0.06$ & $5.4 \pm 1.1$ & 1.332 \\
\hline
\end{tabular}


Rheological properties of the cornea have been studied previously by, for example, Ionescu et al. [36], Petsche et al. [37] and Hatami-Marbini [38]. Due to the differences in the testing conditions, experimental procedures and samples, the results are not directly comparable with each other or with our results.

\subsection{Mechanical properties}

For tissues and hydrogels, the stress-strain curve is non-linear in the elastic portion even at small strains. For this reason, a polynomial fit, instead of a linear fit, was used for the data. Moreover, the stiffness as a function of strain was represented to illustrate the material behavior in a wider strain range. The representative compressive stress as a function of the deformation strain curves are shown in Fig. 3 (c). The results showed that the hydrogels were initially resistant to deformation, but became progressively stiffer as load increased. Finally, the hydrogels fractured at $55 \%$ to $70 \%$ strain range. Due to a higher fracture strain, the $\mathrm{H} 1$ hydrogel was considered to be more elastic compared to the $\mathrm{H} 1 \mathrm{C}$ and $\mathrm{H} 2$ hydrogels, whereas the col I-containing H1C hydrogel was the least elastic.

The curves of average stiffness as a function of increasing strain are shown in Fig. 3 (d). The stiffness was shown to be strain dependent. At low strains (0\% to $20 \%$ ) the stiffness was quite constant, but increased after that more or less depending on the sample. This phenomenon is called strain hardening and it indicates a non-linear deformation characteristic of hydrogels under compression [39].

The second-order elastic constants (stiffness at zero strain) of the hydrogels are shown in Table 2. The H1C hydrogel with highest second-order elastic constant showed significant difference $(p<0.05)$ with respect to the $\mathrm{H} 2$ hydrogel. Otherwise, there were no statistically significant differences between the hydrogels.

In the cornea, the predominant component for its mechanical strength is the stroma [40]. Although, the mechanical properties of the studied hydrogels were not close to the native tissue (0.1 to $57 \mathrm{MPa}$ [41]), the hydrogel films were still fairly easy to handle during the cell culturing. This was also one of the main requirements for the material, more than just replicate the mechanical properties, which might be redundant for cell delivery applications.

\subsection{Optical properties}

The refractive index data of hydrogels was presented as the reflection coefficient, and it was plotted as a function of angle of incidence curves, which are shown in Fig. S5. The angle value was derived by drawing tangent lines on the vertical and horizontal parts of the curve and determining the angle at their intersection point. The calculated refractive indices are shown in Table 2. The refractive indices of all hydrogels were close to that of water $(\mathrm{n}=1.333)$. The $\mathrm{H} 1$ hydrogel had the highest refractive index of the three, bringing it closest to that of the native corneal stroma ( $\mathrm{n}=1.376$ [42]). The rays pass from the 
cornea into the aqueous humor having a lower refractive index $(n=1.336)$, so most of the reflection occurs at the cornea-air interface.

Although cornea represents the strongest part of the refracting power of the eye, the exact replication of its refractive capacity may not be necessary for clinically viable corneal biomaterials. As refractive errors can be easily adjusted with spectacles, the transparency of the material is more important than its correct refractive properties. This is especially true for degradable materials, which should promote the healing of healthy stromal tissue to ultimately regain the refraction power of the cornea.

The visible light transmission spectra of hydrogels are shown in Fig. 4 (a). The light transmittances for $\mathrm{H} 1, \mathrm{H} 1 \mathrm{C}$ and $\mathrm{H} 2$ hydrogels were $85 \pm 1,82 \pm 1$ and $90 \pm 1 \%$, respectively in the $400-700 \mathrm{~nm}$ wavelength range. The transparency of the hydrogels was close to the native cornea (>87\%) [43]. The results showed that $\mathrm{H} 2$ hydrogel was slightly more transparent than H1-based hydrogels. Also, the addition of col I slightly lowered the transparency. The visible inspection of the samples (inside the cuvettes) also supported these findings. While $\mathrm{H} 2$ hydrogel looked visibly clear, the crosslinking was clearly seen inside the H1-based hydrogels. This can be explained with the higher polymer concentration and higher crosslinking density of H1-based hydrogels. The sample thickness used in the transparency measurements was significantly higher than the one intended for the final application. Therefore, thinner hydrogel films were photographed in order to show their optical clarity and transparency. As the photographic images presented in Fig. 4 (b) (and (c)) show, all hydrogels were visibly transparent, even with the addition of col I.

(a)

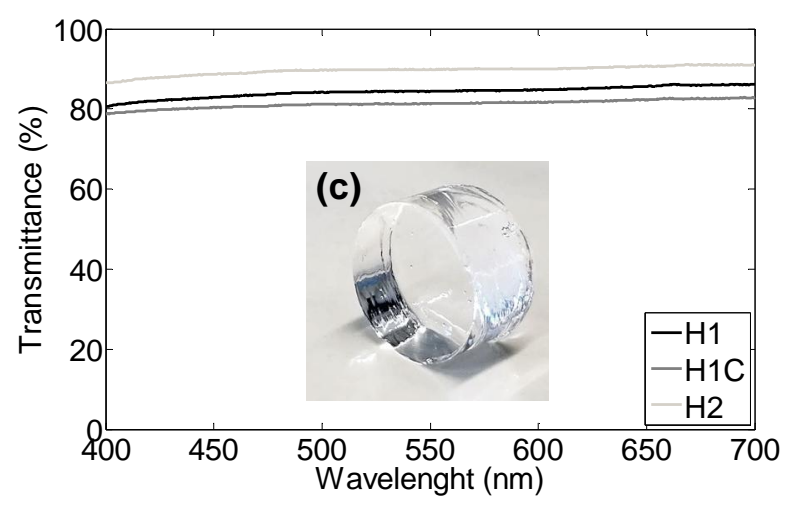

(b)

$\mathrm{H1}$ :

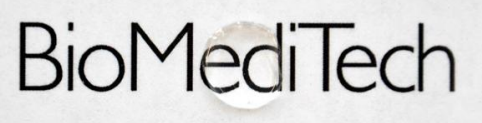

$\mathrm{H} 1 \mathrm{C}$ :

BioMediTech

H2:

Fig. 4. Transparency of HA-based hydrogels. (a) Light transmission spectra of HALD1-HACDH (H1), HALD1-HACDH-col I (H1C) and HAL2-HAADH (H2) hydrogels. (b) Photographic images of H1, $\mathrm{H} 1 \mathrm{C}$ and $\mathrm{H} 2$ hydrogels (diameter $10 \mathrm{~mm} \times$ thickness $1 \mathrm{~mm}$ ) showing their transparency. (c) Photographic image of H1-based hydrogel (diameter $12 \mathrm{~mm} \times$ thickness $7 \mathrm{~mm}$ ) prepared for the compression testing. 
We measured the optical properties from the hydrogel delivery vehicle alone. However, we note that encapsulating cells in the hydrogels causes light scattering, thus affecting their optical properties. Furthermore, both the refractive index and the transparency may change after implantation due to swelling and degradation, as well as the proliferation and differentiation of encapsulated cells.

\subsection{In vitro cell culture}

Human ASCs were encapsulated into the three HA-based hydrogels and their viability was assessed qualitatively using Live/Dead staining and the relative metabolic activity in different hydrogels was analyzed with PrestoBlue ${ }^{\circledR}$. Both the Live/Dead staining (Fig 5 (a)) and the PrestoBlue ${ }^{\circledR}$ results (Fig. 5 (b)) show that all HA-based hydrogels were capable of sustaining short-term cell survival. However, the more unstable $\mathrm{H} 2$ hydrogel samples degraded during the first three days of culture, making their handling for imaging and PrestoBlue ${ }^{\circledR}$ analysis difficult. For this reason, no images could be obtained after the first time point.

Live/Dead staining showed that the number of live cells seemed to decrease from the first time point onwards, although the number of dead cells did not increase. The major cause for this cell loss seems to be cell movement away from the hydrogels, which was observed during culture (Fig. 5 (c)). Additionally, hydrogel swelling, which pulls the cells further away from each other, and possible washout of unattached cells may contribute to apparent cell loss from the hydrogels. The col I-containing H1C hydrogel seemed to support hASC attachment better than the purely HA-based H1 hydrogel, based on the presence of more cells with elongated morphology in the Live/Dead images. Previous research has also shown, that the addition of ECM components, such as col I and laminin to hyaluronan hydrogels is required to support cellular attachment and elongated morphology for cardiomyocytes [44], vocal fold fibroblasts [13] and neural cells [18].

Cell metabolic activity seemed to decrease in all hydrogels after the first time point (Fig. 5 (b)). The trend was similar in all hydrogels, but the onset of the steep decline in metabolic activity varied; for the $\mathrm{H} 2$ hydrogel, the drop occurred between day 1 and day 3, whereas for $\mathrm{H} 1$ and $\mathrm{H} 1 \mathrm{C}$ it occurred between day 3 and day 7 . The decline in cell metabolic activity correlated with the observed decrease of cells in the Live/Dead images, also indicating that cells escaped from the hydrogels. However, the remaining cells maintained their metabolic activity at a steady level for the rest of the culture period. Importantly, the H1C hydrogel-encapsulated hASCs had significantly higher metabolic activity than those in the H1 hydrogel in the two last time points, which indicates that addition of col I has a significant effect on promoting active cell metabolism and survival.

The Live/Dead and PrestoBlue ${ }^{\circledR}$ results indicate that hASCs survive encapsulation into all of the HAhydrogels, but the addition of col I is required for cell attachment to the hydrogel matrix. The efficient loss of cells from the hydrogels consisting of purely HA-components, suggests they could be suitable cell delivery vehicles, with either a rapid (H2) or more delayed delivery (H1) of hASCs to stromal 
defects. However, for long-term tissue integration of the hydrogel matrix, cell attachment may be required for efficient cell proliferation and ECM synthesis [45].



Fig 5. Cell viability and metabolic activity in HA-based hydrogels. (a) Live-dead staining of hASCs encapsulated in HALD1-HACDH (H1), HALD1-HACDH-col I (H1C) and HALD2-HAADH (H2) hydrogels at different time points. Live cells are shown in green and dead cells in red, with arrows pointing at elongated cells. (b) Metabolic activity of hydrogel-encapsulated hASCs during in vitro culture as measured by PrestoBlue ${ }^{\circledR}$ analysis. The bars show the relative fluorescence values of cellladen hydrogel samples with mean and standard deviation. The $\mathrm{H} 2$ hydrogel could not be handled for imaging any more at day 3 , and the PrestoBlue ${ }^{\circledR}$ analysis could not be performed after that time point. *significant difference at $p<0.05$ (c) Representative image of the observed cell growth out of the hydrogels and under the PDMS mold at day 9. Scale bars in the images are $200 \mu \mathrm{m}$.

\subsection{Implantation to corneal organ culture model}

The corneal organ culture model is an excellent method to study cellular interactions at the organ level and to evaluate the clinical feasibility of biomaterials for corneal applications [46,47]. The corneal organ culture model has been previously used mainly to study re-epithelialization, since the organ-cultured 
corneas retain their capability for epithelial regeneration for as long as two months in culture [28]. Although the results obtained from the corneal organ culture model always require validation in an in vivo animal model, its use in fast and low-cost preliminary screening of corneal biomaterials can significantly reduce unnecessary animal testing.

We used excised porcine corneas to model the delivery of hASCs in the HA-based hydrogels into stromal defects. As a proof-of-concept, we inflicted large stromal wounds, into which the hydrogels were implanted with and without hASCs. The immunohistological evaluation of the porcine corneas with hydrogel-delivered hASCs revealed cell migration into the corneal stroma, after just one week of culture (Fig. 6). Successful integration of hASCs into the stroma was observed for all of the hydrogels, although H1C hydrogel samples seemed to show slightly less migrated hASCs in the stroma than H1 and H2. Possibly, collagen's effect on improving hASC attachment may have delayed their migration to the stroma from the $\mathrm{H} 1 \mathrm{C}$ hydrogel. The integration of hASCs to the corneal stroma in the organ culture model demonstrates the clinical feasibility of the hydrogels for stromal delivery of hASCs.

Despite the predominant loss of the hydrogels themselves during histological processing, reepithelialization of the corneal surface was evident from the tissue sections. This was clearly seen in the vehicle-only controls of $\mathrm{H} 1$ and $\mathrm{H} 2$ in Fig. 6 (j) and (m), respectively. Fig. 6 (d) also implies epithelial outgrowth over the $\mathrm{H} 1 \mathrm{C}$ hydrogel sample, although the complete epithelium is not visible in the section. Previous research has also reported that HA can support corneal epithelial cell growth [48], which further validates its use in the repair of the underlying stroma. However, some sections showed epithelial growth at the edges of the wound rather than on top of the hydrogel (shown in Fig. $6(\mathrm{k})$ ), indicating that the gels were not always in good contact with the underlying tissue.

The organ culture model also revealed that the degradation rate of the hydrogels on the corneal surface is not directly comparable to the dilute medium environment of the swelling tests or in vitro culture. For example, the complete re-epithelialization and shape retention of the corneal surface in Fig. $6(\mathrm{~m})$ indicates that the $\mathrm{H} 2$ hydrogel remained stable for the entire culture period of seven days, whereas during in vitro culture, the hydrogel could no longer be observed after three days. 


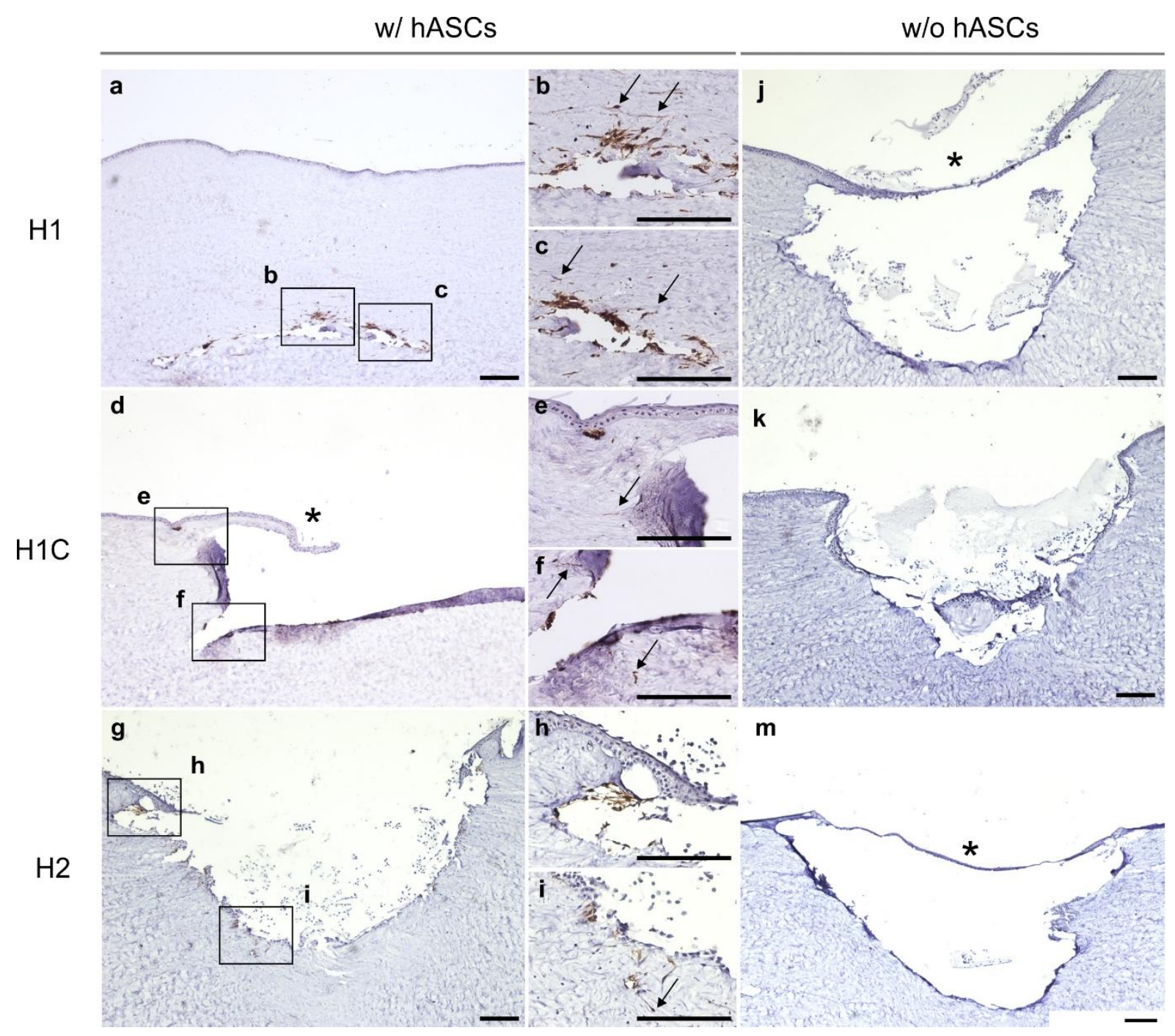

Fig. 6. Immunohistochemical detection of hydrogel-delivered hASCs after seven days in porcine corneal organ culture model using human cell marker TRA-1-85. Images on the left panel (a-i) describe hydrogels HALD1-HACDH (H1), HALD1-HACDH-col I (H1C) and HALD2-HAADH (H2) with encapsulated hASCs, and the images on the right $(\mathbf{j}-\mathbf{m})$ depict delivery of each gel vehicle only. All other sections depict the central wound area, except that of H1 w/ hASCs, which was sectioned near the wound edge. Successful hASC integration into the corneal stroma was seen for all hydrogels (examples marked with arrows), and epithelium growth on top of the transplanted gels was observed with all gels, even without hASCs (denoted by * in images $\mathbf{e}, \mathbf{j}$ and $\mathbf{m}$ ). However, epithelial overgrowth could not be verified for all samples, as the gels were lost during histological processing. Scale bars in the images are $200 \mu \mathrm{m}$.

\section{Conclusions}

In this study, we fabricated two types of hydrazone crosslinked HA-based hydrogels, with the addition of human collagen I. We characterized the physical and mechanical properties of these hydrogels and evaluated their potential as cell delivery vehicles to the corneal stroma. Based on the results, HALD1- 
HACDH hydrogels showed better stability and manageability compared to the HALD2-HAADH hydrogel, which degraded in vitro in three days. These hydrogels had also good optical properties, the refractive indices were close to the native cornea and they were visually transparent. Although all hydrogels showed good hASC survival directly after encapsulation, only the col I-containing HALD1HACDH-col I hydrogel showed cells with elongated morphology, and significantly higher cell metabolic activity than the HALD1-HACDH gel. Corneal organ culture model suggests that these hydrogels could be used as injectable cell delivery vehicles to corneal stromal defects, allowing efficient cell integration to the stroma and overgrowth of epithelial cells. Biodegradability of the HA components and favorable properties of hydrazone crosslinking, such as short gelation time, lack of harmful reagents or side-products and mild reaction conditions, together with the results make these hydrogels a potential material for hASC delivery to treat corneal stromal defects.

\section{Acknowledgements}

This work was funded by TEKES (the Finnish Funding Agency for Innovation) Human Spare Parts project and the European COST Action BM-1302 Joining forces in corneal regeneration research. The funding sources were not involved in conduction if the research or interpretation of the results. The authors would like to thank Ph.D. Alexandre Efimov and Laboratory Attendant Anne-Maarit Tikkanen (Faculty of Natural Sciences, Laboratory of Chemistry and Bioengineering, Tampere University of Technology, Tampere, Finland) for their help related to the NMR- and FTIR-measurements. We would also like to thank Ph.D. Maria Notara (University Hospital Cologne, Cologne, Germany) for expertise in the corneal organ culture method, Marja-Leena Koskinen for aiding with the histological analysis and Miia Juntunen for technical assistance and the microscopy core facility of University of Tampere for the tools and expertise in cell imaging.

\section{Appendix A. Supplementary Material}

\section{References}

[1] WHO, Priority eye diseases, 2017 (2016).

[2] CE Ghezzi, J Rnjak-Kovacina, DL Kaplan. Corneal tissue engineering: recent advances and future perspectives, Tissue Engineering Part B: Reviews. 21 (2015) 278-287.

[3] JY Oh, MK Kim, MS Shin, HJ Lee, JH Ko, WR Wee, et al. The anti-inflammatory and antiangiogenic role of mesenchymal stem cells in corneal wound healing following chemical injury, Stem Cells. 26 (2008) 1047-1055.

[4] SK Mittal, M Omoto, A Amouzegar, A Sahu, A Rezazadeh, KR Katikireddy, et al. Restoration of Corneal Transparency by Mesenchymal Stem Cells, Stem cell reports. 7 (2016) 583-590.

[5] B Lindroos, R Suuronen, S Miettinen. The potential of adipose stem cells in regenerative medicine, Stem Cell Reviews and Reports. 7 (2011) 269-291. 
[6] F Arnalich-Montiel, S Pastor, A Blazquez-Martinez, J Fernandez-Delgado, M Nistal, JL Alio del Barrio, et al. Adipose-Derived Stem Cells Are a Source for Cell Therapy of the Corneal Stroma, Stem Cells. 26 (2008) 570-579.

[7] L Espandar, B Bunnell, GY Wang, P Gregory, C McBride, M Moshirfar. Adipose-Derived Stem Cells on Hyaluronic Acid-Derived Scaffold: A New Horizon in Bioengineered Cornea, Arch.Ophthalmol. 130 (2012) 202-208.

[8] J Alio del Barrio, M Chiesa, N Garagorri, N Garcia-Urquia, J Fernandez-Delgado, L Bataille, et al. Acellular human corneal matrix sheets seeded with human adipose-derived mesenchymal stem cells integrate functionally in an experimental animal model, Exp.Eye Res. 132 (2015) 91-100.

[9] KM Meek, C Knupp. Corneal structure and transparency, Prog.Retin.Eye Res. 49 (2015) 1-16.

[10] J Fraser, T Laurent, U Laurent. Hyaluronan: its nature, distribution, functions and turnover, J.Intern.Med. 242 (1997) 27-33.

[11] X Xu, AK Jha, DA Harrington, MC Farach-Carson, X Jia. Hyaluronic acid-based hydrogels: from a natural polysaccharide to complex networks, Soft matter. 8 (2012) 3280-3294.

[12] Y Jiang, J Chen, C Deng, EJ Suuronen, Z Zhong. Click hydrogels, microgels and nanogels: emerging platforms for drug delivery and tissue engineering, Biomaterials. 35 (2014) 4969-4985.

[13] X Jia, Y Yeo, RJ Clifton, T Jiao, DS Kohane, JB Kobler, et al. Hyaluronic acid-based microgels and microgel networks for vocal fold regeneration, Biomacromolecules. 7 (2006) 3336-3344.

[14] E Martínez-Sanz, DA Ossipov, J Hilborn, S Larsson, KB Jonsson, OP Varghese. Bone reservoir: injectable hyaluronic acid hydrogel for minimal invasive bone augmentation, J.Controlled Release. 152 (2011) 232-240.

[15] P Bulpitt, D Aeschlimann. New strategy for chemical modification of hyaluronic acid: preparation of functionalized derivatives and their use in the formation of novel biocompatible hydrogels, Journal of Biomedical Materials Research Part A. 47 (1999) 152-169.

[16] J Karvinen, JT Koivisto, I Jönkkäri, M Kellomäki. The production of injectable hydrazone crosslinked gellan gum-hyaluronan-hydrogels with tunable mechanical and physical properties, Journal of the Mechanical Behavior of Biomedical Materials. 71 (2017) 383-391.

[17] OP Oommen, S Wang, M Kisiel, M Sloff, J Hilborn, OP Varghese. Smart design of stable extracellular matrix mimetic hydrogel: synthesis, characterization, and in vitro and in vivo evaluation for tissue engineering, Advanced Functional Materials. 23 (2013) 1273-1280.

[18] S Suri, CE Schmidt. Cell-laden hydrogel constructs of hyaluronic acid, collagen, and laminin for neural tissue engineering, Tissue Engineering Part A. 16 (2010) 1703-1716.

[19] DA Ossipov, S Piskounova, J Hilborn. Poly (vinyl alcohol) cross-linkers for in vivo injectable hydrogels, Macromolecules. 41 (2008) 3971-3982.

[20] KH Bouhadir, DS Hausman, DJ Mooney. Synthesis of cross-linked poly (aldehyde guluronate) hydrogels, Polymer. 40 (1999) 3575-3584.

[21] ASTM Standard F2900:2011, Standard Guide For Charachterization of Hydrogels used in Regerative Medicine, (2011). 
[22] K Brugger. Thermodynamic definition of higher order elastic coefficients, Physical Review. 133 (1964) A1611.

[23] JF Nye, Physical properties of crystals: their representation by tensors and matrices, Oxford University Press 1985.

[24] WD Callister, DG Rethwich, Materials science and engineering: an introduction, 7th ed., Wiley, New York, 2007.

[25] H Nakajima, APPENDIX B: Table of Refractive Indices for BK7, Optical Design Using Excel: Practical Calculations for Laser Optical Systems, 1st ed., John Wiley \& Sons Singapore Pte Ltd, 2015, pp. 277-278.

[26] JM Gimble, F Guilak. Adipose-derived adult stem cells: isolation, characterization, and differentiation potential, Cytotherapy. 5 (2003) 362-369.

[27] B Lindroos, S Boucher, L Chase, H Kuokkanen, H Huhtala, R Haataja, et al. Serum-free, xenofree culture media maintain the proliferation rate and multipotentiality of adipose stem cells in vitro, Cytotherapy. 11 (2009) 958-972.

[28] M Notara, S Schrader, JT Daniels. The porcine limbal epithelial stem cell niche as a new model for the study of transplanted tissue-engineered human limbal epithelial cells, Tissue Engineering Part A. 17 (2011) 741-750.

[29] W Lin, S Shuster, HI Maibach, R Stern. Patterns of hyaluronan staining are modified by fixation techniques, Journal of Histochemistry \& Cytochemistry. 45 (1997) 1157-1163.

[30] DA Ossipov, S Piskounova, OP Varghese, J Hilborn. Functionalization of hyaluronic acid with chemoselective groups via a disulfide-based protection strategy for in situ formation of mechanically stable hydrogels, Biomacromolecules. 11 (2010) 2247-2254.

[31] C García-González, M Alnaief, I Smirnova. Polysaccharide-based aerogels-Promising biodegradable carriers for drug delivery systems, Carbohydr.Polym. 86 (2011) 1425-1438.

[32] SL Wilson, AJ El Haj, Y Yang. Control of scar tissue formation in the cornea: strategies in clinical and corneal tissue engineering, Journal of functional biomaterials. 3 (2012) 642-687.

[33] ER Morris, K Nishinari, M Rinaudo. Gelation of gellan-a review, Food Hydrocoll. 28 (2012) 373-411.

[34] MJ Moura, MM Figueiredo, MH Gil. Rheological study of genipin cross-linked chitosan hydrogels, Biomacromolecules. 8 (2007) 3823-3829.

[35] A Borzacchiello, L Ambrosio, Structure-property relationships in Hydrogels, Hydrogels, Springer, 2009, pp. 9-20.

[36] A Ionescu, M Alaminos, de la Cruz Cardona, Juan, Durán, Juan de Dios García-López, M González-Andrades, R Ghinea, et al. Investigating a novel nanostructured fibrin-agarose biomaterial for human cornea tissue engineering: Rheological properties, Journal of the mechanical behavior of biomedical materials. 4 (2011) 1963-1973.

[37] SJ Petsche, D Chernyak, J Martiz, ME Levenston, PM Pinsky. Depth-dependent transverse shear properties of the human corneal stroma, Invest.Ophthalmol.Vis.Sci. 53 (2012) 873-880. 
[38] H Hatami-Marbini. Viscoelastic shear properties of the corneal stroma, J.Biomech. 47 (2014) 723-728.

[39] G Lamouche, BF Kennedy, KM Kennedy, C Bisaillon, A Curatolo, G Campbell, et al. Review of tissue simulating phantoms with controllable optical, mechanical and structural properties for use in optical coherence tomography, Biomedical optics express. 3 (2012) 1381-1398.

[40] WJ Dupps, SE Wilson. Biomechanics and wound healing in the cornea, Exp.Eye Res. 83 (2006) 709-720.

[41] N Garcia-Porta, P Fernandes, A Queiros, J Salgado-Borges, M Parafita-Mato, JM GonzálezMéijome. Corneal biomechanical properties in different ocular conditions and new measurement techniques, ISRN ophthalmology. 2014 (2014).

[42] KM Meek, S Dennis, S Khan, Changes in the Refractive Index of the Stroma and Its Extrafibrillar Matrix When the Cornea Swells, Biophysical Journal. 85 (2003) 2205-2212.

[43] W Liu, C Deng, CR McLaughlin, P Fagerholm, NS Lagali, B Heyne, et al. Collagenphosphorylcholine interpenetrating network hydrogels as corneal substitutes, Biomaterials. 30 (2009) 1551-1559.

[44] J Dahlmann, A Krause, L Möller, G Kensah, M Möwes, A Diekmann, et al. Fully defined in situ cross-linkable alginate and hyaluronic acid hydrogels for myocardial tissue engineering, Biomaterials. 34 (2013) 940-951.

[45] J Zhu, RE Marchant. Design properties of hydrogel tissue-engineering scaffolds, Expert review of medical devices. 8 (2011) 607-626.

[46] MDM Evans, GA McFarland, RZ Xie, S Taylor, JS Wilkie, H Chaouk. The use of corneal organ culture in biocompatibility studies, Biomaterials. 23 (2002) 1359-1367.

[47] S Sandeman, A Lloyd, B Tighe, V Franklin, J Li, F Lydon, et al. A model for the preliminary biological screening of potential keratoprosthetic biomaterials, Biomaterials. 24 (2003) 4729-4739.

[48] JA Gomes, R Amankwah, A Powell-Richards, HS Dua. Sodium hyaluronate (hyaluronic acid) promotes migration of human corneal epithelial cells in vitro, Br.J.Ophthalmol. 88 (2004) 821-825. 